\title{
Operação de Blalock-Taussig modificada para o tratamento paliativo de cardiopatias congênitas com hipofluxo pulmonar
}

Miguel A. MALUF * , José Carlos S. ANDRADE *, Antônio CARVALHO *, Roberto CATANI *, Hermínio VEGA * , José L. ANDRADE ${ }^{\star}$, Célia SILVA * , Werther B. CARVALHO * ${ }^{*}$ Énio BUFFOLO *

MALUF, M. A.; ANDRADE, J. C. S.; CARVALHO, A.; CATANI, R.; VEGA, H.; ANDRADE, J. L.; SILVA, C.; CARVALHO, W. B.; BUFFOLO, E. - Operação de Blalock-Tassig modificada para o tratamento paliativo de cardiopatias congênitas com hipofluxo pulmonar. Rev. Bras. Cir. Cardiovasc., 10 (3): $126-132,1995$.

RESUMO: De janeiro de 1990 a novembro de 1994, 72 pacientes portadores de cardiopatias congênitas com hipofluxo pulmonar foram submetidos à operaçấo de Blalock-Taussig. A idade variou entre 2 dias e 11 anos (M:9,0 meses); $44(61,1 \%)$ eram do sexo masculino e $28(38,8 \%)$ do feminino; $38(52,8 \%)$ casos portadores de tétrade de Fallot: $7(9.7 \%)$ atresia pulmonar com septo interventricular integro (AP c/ SIVI); $6(8,4 \%)$ transposiçăo das grandes artérias (TGA) + estenose pulmonar $(E P) ; 6(8,4 \%)$ atresia tricúspide (AT) + EP; $6(8,4 \%)$ dupla via de entrada (DVE) do ventriculo direito (VD) ou ventriculo esquerdo (VE) + (EP); $3(4,2 \%)$ transposiçăo corrigida das grandes artérias (TCGA) + comunicaçāo interventricular (CIV) + EP; $2(2,7 \%)$ dupla via de saida (DVS) de VD ou VE + EP; $2(2,7 \%)$ defeito septal atrioventricular $(D S A V)+E P ; 2(2,7 \%)$ isomerismo direito (D) ou (E) + EP. Os critérios de indicaçāo foram: a) neonatos com cardiopatias "ducto dependente"; b) lactentes com piora de cianose ou crise de hipóxia; c) crianças durante a infảncia com cardiopatias sem chance de uma correçāo biventricular. A técnica empregada foi a operaçāo de Blalock-Taussig modificada, interpondo prótese entre artéria subclávia e artéria pulmonar. A prótese de Polytetrafluoroethylene (PTFE) foi usada em $69(94,5 \%)$ casos, veia umbilical em $3(4,1 \%)$ casos e artéria mamária bovina em $1(1,4 \%)$ caso. Durante 0 ato operatório foi administrada dose única de heparina ( $1 \mathrm{mg} \times \mathrm{kg}$ peso), năo sendo neutralizada no pós-operatório $(\mathrm{PO})$ e no seguimento tardio, dispensado o uso de anticoagulantes ou antiagregantes plaquetários. Houve $8(11,1 \%)$ óbitos no PO imediato: $5(6,9 \%)$ casos por obstruçāo da prótese ( 3 foram reoperados), $2(2,7 \%)$ casos por morte súbita (AP c/ SIVI) e $1(1,3 \%)$ devido a processo infeccioso pulmonar. A obstruçăo da prótese como principal causa de óbito esteve diretamente relacionada à anatomia dos vasos (calibre da artéria subclávia e pulmonar) e ou problemas de técnica cirúrgica. A operaçăo de Blalock-Taussig modificada demonstrou ser um método confiável e seguro no tratamento paliativo das cardiopatias com hipofluxo puimonar.

DESCRITORES: Blalock-Taussig, operação de, modificada. Cardiopatias congênitas, operaçāo de Blalock - Taussig. Cardiopatias congênitas, hipofluxo pulmonar, cirurgia.

\footnotetext{
Trabalho realizado na Disciplina de Cirurgla Cardiovascular da Escola Paulista de Medicina da Universidade Federal de São Paulo. São Paulo, SP, Brasil.

Apresentado ao $22^{2}$ Congresso Nacional de Cirurgia Cardiaca. Brasilia, DF, 30 de março a $1^{2}$ de abril, 1995.

- Da Escola Paulista de Medicina de Universidade de Sáo Paulo.

Endereço para correspondencia: Miguel A. Malut. Disciplina de Cirurgia Cardiovascular. Escola Paulista de Medicina. Rua Napoleầo de Barros, 715

. $3^{2}$ andar. CEP 04024-002 Săo Paulo, SP, Brasili. Tel. (011) 571-2719 - Fax (011) 571-8785.
} 
MALUF, M. A.; ANDRADE, J. C. S.; CARVALHO, A.; CATANI, R.; VEGA, H.; ANDRADE, J. L.; SILVA, C.; CARVALHO, W. B.; BUFFOLO, E. - Operaçāo de Blalock-Tassig modificada para o tratamento paliativo de cardiopatias congênitas com hipofluxo pulmonar. Rev. Bras. Cir. Cardiovasc., 10 (3): 126-132, 1995.

\section{INTRODUÇĀO}

Apesar dos recentes avanços na correção cirúrgica das cardiopatias congênitas com hipofluxo pulmonar no $1^{\circ}$ ano de vida, existe, ainda, um número importante de pacientes onde o risco de uma correção anatômica ou funcional é muito alto nesta faixa etária. Este grupo de pacientes pode ser beneficiado com a realização de uma derivação sistêmico-pulmonar, permitindo o seu desenvolvimento pondo-estatural com diminuiçăo dos sintomas até a correçăo definitiva.

A operação de Blalock-Taussig ${ }^{3}$ (BT) constitui uma boa opçăo tática para este grupo de pacientes. $\mathrm{O}$ uso de próteses de Polytetrafluoroethylene (PTFE) tem permitido a realização da operação em artérias pulmonares de fino calibre, com resultados satisfatórios (BT modificado) $5,7,10$.

As principais vantagens da operação de BT modificada são:

- Facilidade técnica na sua realização.

- Fluxo pulmonar regulado pela artéria subclávia.

- Preservação da integridade da artéria subclávia.

- Perviabilidade a longo prazo.

- Facilidade técnica da interrupção do fluxo durante a correção definitiva da anomalia.

- Possibilidade de escolha da via e lado de acesso (direito ou esquerdo), para a realização da operação.

- Uso do tronco braquiocefálico nos casos de artéria subclàvia de pequeno calibre.

\section{CASUISTICA E MÉTODOS}

Setenta e dois pacientes portadores de cardiopatias congênitas com hipofluxo pulmonar foram submetidos à operação de Blalock-Taussig modificada, de janeiro de 1990 a novembro de 1994 , na Disciplina de Cirurgia Cardiovascular da Escola Paulista de Medicina.

$A$ idade variou entre 2 dias e 11 anos (M:9,0 meses); onde $33(45,84 \%)$ casos tinham menos de 1 mês; $44(61,1 \%)$ eram do sexo masculino e 28 $(38,8 \%)$ do feminino; $38(52,8 \%)$ casos portadores de tétrade de Fallot; $7(9,7 \%)$ atresia pulmonar com septo interventricular integro (AP c/ SIVI); $6(8,4 \%)$ transposição das grandes artérias (TGA) + estenose pulmonar (EP); $6(8,4 \%)$ atresia tricúspide (AT) + EP; $6(8,4 \%)$ dupla via de entrada (DVE) do ventrículo direito (VD) ou ventriculo esquerdo (VE) + EP; 3 $(4,2 \%)$ transposição corrigida das grandes artérias (TCGA) +comunicação interventricular (CIV) + EP; 2 $(2,7 \%)$ dupla via de saída (DVS) do VD ou VE + EP: $2(2,7 \%)$ defeito septal atrioventricular (DSAV) + EP: $2(2,7 \%)$ isomerismo direito (D) ou (E) + EP (Tabela 1).

A conduta e os critérios de indicação neste grupo de pacientes obedeceu a:

1 Neonatos com cardiopatias "ducto dependente", tratados com prostaglandina $E_{1}\left(P G E_{1}\right)$.

2 Lactentes com piora do quadro clínico (cianose, crises de hipóxia), a maioria tratada com $\beta$-bloqueadores.

3 Crianças durante a infância, portadoras de cardiopatias sem possibilidade de correçăo biventricular (ventrículo único funcional).

TABELA 1

PACIENTES SUBMETIDOS A OPERAÇÃO DE BLALOCK-TAUSSIG MODIFICADO - 1990-1994

\begin{tabular}{lcccc}
\hline $\begin{array}{l}\text { Anomalias } \\
\text { Cardiacas }\end{array}$ & $\begin{array}{c}\text { Com Estenose } \\
\text { Pulmonar }\end{array}$ & $\begin{array}{c}\text { Com Atresia } \\
\text { Pulmonar }\end{array}$ & Total \\
\hline T. Fallot & 18 & 20 & 38 & 52,8 \\
AP cl SIVI & - & 7 & 7 & 9,7 \\
TGA + EP & 4 & 2 & 6 & 8,4 \\
AT & 6 & 3 & 6 & 8,4 \\
DVEV + EP & 3 & - & 3 & 2 \\
TCGA + EP & 3 & - & 2 & 2,2 \\
DVSV + EP & 2 & - & 2 & 2,7 \\
DSA-V + EP & 2 & - & 2,7 \\
Isomerismo & 2 & 32 & 72 & 100 \\
\hline Total & 40 & & 2 & \\
\hline
\end{tabular}

T Fallot: tétrade de Fallot; AP of SIVI: atresia pulmonar com septo interventricular integro: TGA: transposiçāo das grandes artérias; EP: estenose pulmonar; AT: atresia tricúspide; DVEV: dupla via de entrada ventricular; DVSV: dupla via de saída ventricular; TCGA; transposiçāo corrigida das grandes artérias. DSA-V: defeito septal atrioventricular. 
MALUF, M. A.; ANDRADE, J. C. S.; CARVALHO, A.; CATANI, R.; VEGA, H.; ANDRADE, J. L.; SILVA, C.; CARVALHO, W. B.; BUFFOLO, E. - Operação de Blalock-Tassig modificada para o tratamento paliativo de cardiopatias congênitas com hipofluxo pulmonar. Rev. Bras. Cir. Cardiovasc., 10 (3): 126-132, 1995.

TABELA 2

OPERAÇĀO DE BLALOCK-TAUSSIG MODIFICADA

\begin{tabular}{|c|c|c|c|c|c|c|c|c|c|}
\hline \multirow[t]{2}{*}{$\begin{array}{l}\text { Tipo de } \\
\text { abordagem }\end{array}$} & \multicolumn{2}{|c|}{$\begin{array}{c}\text { Anastomose } \\
\text { com }\end{array}$} & \multicolumn{3}{|c|}{ Tipo de Prótese } & \multicolumn{2}{|c|}{$\begin{array}{c}\text { Diâmetro } \\
\text { Prófese }\end{array}$} & \multicolumn{2}{|c|}{ Total } \\
\hline & AS. & TBC & PTFE & $v U$ & $A M B$ & $4 \mathrm{~mm}$ & $5 \mathrm{~mm}$ & $N^{9}$ & $\%$ \\
\hline Toracotomia D & 35 & 25 & 60 & - & - & 2 & 58 & 60 & 83,3 \\
\hline Toracotomia E & 12 & $=$ & 8 & 3 & 1 & 1 & 11 & 12 & 16,7 \\
\hline Total & 47 & 25 & 68 & 3 & 1 & 3 & 69 & 72 & 100 \\
\hline
\end{tabular}

D: direita; E: esquerda; AS: artéria subclávia; TBC: tronco braquiocefálico; VU: veia umbilical; AMB: artéria mamária bovina; PTFE: Polytetrafluoroethylene.

\section{TÉCNICA CIRÚRGICA}

Com o paciente intubado e devidamente monitorizado: eletrocardiograma, oximetria pulsátil continua, pressăo arterial média, pressăo venosa central e controle de diurese, foi posicionado na mesa de operaçōes. A abordagem foi a direita em $60(83,3 \%)$ casos e a esquerda em $12(16,7 \%)$.

Após abertura do tórax pelo $3^{\circ}$ espaço intercostal e afastado o pulmão cuidadosamente, foram dissecados artéria subclávia e o tronco braquiocefálico, iniciada na porçăo mais visivel em direção ao mediastino, afastando-se do nervo frênico.

A seguir foi dissecada a artéria pulmonar, cadarçados os seus ramos, cuidando de não realizar qualquer traçăo desnecessária que poderia distorcer o canal arterial e comprometer o fluxo pulmonar, em especial nos casos de atresia pulmonar.

Em todos os casos foi administrada dose única de heparina (1 $\mathrm{mg} \times \mathrm{kg}$ peso), sendo que em nenhum foi neutralizada com protamina.

As próteses empregadas foram: Polytetrafluoroethylene (PTFE) em $68(94,1 \%$ ) casos, veia umbilical em $4(5,5 \%)$ casos e artéria mamária bovina em
$1(1,3 \%)$ caso. Os diâmetros das próteses foram: 5 $\mathrm{mm}$ em $69(95,8 \%)$ casos e $4 \mathrm{~mm}$ em $3(4,1 \%)$ casos. A anastomose foi realizada empregando a artéria subclávia em $47(65,2 \%)$ casos (Tabela 2).

Em $25(34,7 \%)$ crianças menores de 15 dias, que apresentavam artéria subclávia fina, foi usado - tronco braquiocefálico (TBC) para realizar a anastomose (Tabela 3) (Figura 1).

A anastomose da prótese foi realizada mediante sutura contínua, empregando fio Polypropylene 7.0. Atualmente, está sendo usado o fio PTFE 7.0, que reduz a chance de sangramento da prótese na linha de sutura (Figura 2).

Após liberação dos vasos, o pulmăo foi insuflado para desfazer as atelectasias e monitorização da saturação arterial. A presença de frêmito no local da anastomose e a elevação da saturação arterial acima de $80 \%$ foi sinal de funcionamento adequado da derivação.

Após drenagem e fechamento do tórax, as crianças foram conduzidas para a Unidade de Terapia Intensiva Pediátrica, onde permaneceram intubadas e sob ventilaçăo mecânica até a estabilização hemodinåmica.

TABELA 3

OPERAÇĀO DE BLALOCK-TAUSSIG MODIFICADA EMPREGANDO O TRONCO BRAQUIOCEFÁLICO

\begin{tabular}{|c|c|c|c|c|c|c|c|c|c|}
\hline \multirow[t]{2}{*}{ Dlagnóstico } & \multirow{2}{*}{$\begin{array}{c}\text { Casos } \\
N^{\circ}\end{array}$} & \multirow[b]{2}{*}{$\%$} & \multirow[t]{2}{*}{ Idade } & \multirow[b]{2}{*}{ Tipo } & \multirow{2}{*}{\multicolumn{2}{|c|}{$\begin{array}{l}\text { Prótese } \\
\text { Diâmetro }\end{array}$}} & \multirow[b]{2}{*}{$\begin{array}{c}\text { Bom } \\
N^{2}\end{array}$} & \multicolumn{2}{|c|}{ Resultados } \\
\hline & & & & & & & & $\%$ & $\begin{array}{c}\text { Obitos } \\
\text { № \% Causas }\end{array}$ \\
\hline T. Fallot & 8 & (32) & $<15 d$ & PTFE & 8 & & 7 & $(87,5)$ & $1(12,5)$ Inf. Pul. \\
\hline AP c/SIVI & 6 & (24) & $<15 \mathrm{~d}$ & PTFE & 5 & 1 & 5 & $(83,3)$ & $1(16,6)$ M. Súb. \\
\hline AT & 3 & $(12)$ & $<15 \mathrm{~d}$ & PTFE & 3 & & 3 & $(100)$ & 0 \\
\hline Isomerismo & 3 & $(12)$ & $<15$ d & PTFE & 2 & 1 & 3 & $(100)$ & $0-$ \\
\hline DVSV+EP & 2 & (08) & $<15 d$ & PTFE & 2 & & 2 & $(100)$ & $0-$ \\
\hline DVEV+EP & 2 & $(08)$ & $<15 d$ & PTFE & 2 & & 2 & $(100)$ & 0 \\
\hline $\mathrm{TGA}+\mathrm{CIV}+\mathrm{EP}$ & 1 & $(04)$ & $<15 \mathrm{~d}$ & PTFE & 1 & & 1 & $(100)$ & $0-$ \\
\hline Total & 25 & $(100)$ & & & & & 23 & (92) & $2(8)$ \\
\hline
\end{tabular}

T. Fallot: tétrade de Fallot; AP c/ SIVI: atresia pulmonar com septo interventricular íntegro; AT: atresia tricúspide; DVSV: dupla via de saida ventricular; EP: estenose pulmonar; DVEV: dupla via de entrada ventricular; TGA: transposição das grandes artérias; CIV: comunicaçăo interventricular; Inf. Pul.: infecçăo pulmonar; M. Súb: morte súbita. 
MALUF, M. A.; ANDRADE, J. C. S.; CARVALHO, A.; CATANI, R.; VEGA, H.; ANDRADE, J. L.; SILVA, C.; CARVALHO, W. B.; BUFFOLO, E. - Operaçăo de Blalock-Tassig modificada para o tratamento paliativo de cardiopatias congênitas com hipofluxo pulmonar. Rev. Bras. Cir. Cardiovasc., 10 (3): 126-132, 1995.

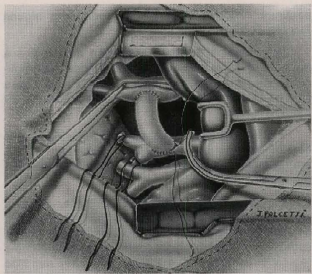

Fig. 1. Desenhodo procedimento cirúrgico. Pinçamento arteriotomia longitudinal do tronco braquiocetálico (TBC); in icio de sutura da prótese de Polytetrafluorethylene (PRFE).

\section{RESULTADOS}

Houve $8(11,1 \%)$ óbitos no pós-operatório imediato, $5(6,9 \%)$ casos evoluiram com queda da saturaçăo, crises de hipóxia e alteraçōes metabólicas; ao exame clínico, nāo foi possivel auscultar sopro que comprovasse a perviabilidade da derivação; 0 ecoDopplercardiograma constatou ausência de fluxo através da prótese e sinais de trombose.

Três crianças foram reoperadas e submetidas a nova derivação sistêmico-pulmonar contralateral. Nenhum paciente sobreviveu.

Duas $(2,7 \%)$ crianças portadoras de AP c/ SIVI tiveram morte súbita no $1^{\circ}$ e $6^{\circ} \mathrm{PO}$, respectivamente.

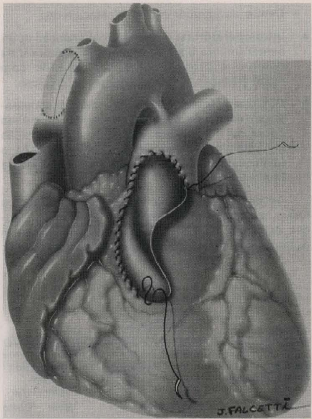

Fig. 2- Desenho do procedimento cirúrgico. Prótese de PTFE implantada entre TBC e artéria pulmonar direita (APD).

O exame ecoDopplercardiográfico pré-operatório mostrou hipoplasia importante do ventrículo direito. Uma $(1,3 \%)$ criança evoluiu com quadro de insuficiência respiratória devido a infecção pulmonar, vindo a falecer no $25^{\circ}$ PO (Tabela 4 ).

TABELA 4

PACIENTES QUE EVOLUIRAM PARA OBITO NO POSS-OPERATÓRIO IMEDIATO

\begin{tabular}{|c|c|c|c|c|c|c|}
\hline $\begin{array}{l}\text { Anomalias } \\
\text { Cardfaca }\end{array}$ & Idade & $\begin{array}{c}\text { Diåmetro } \\
\text { da } \\
\text { Prótese }\end{array}$ & $\begin{array}{c}\text { Tipo } \\
\text { de } \\
\text { Prótese }\end{array}$ & $\begin{array}{c}\text { Intercorrências } \\
\text { no POI }\end{array}$ & $\begin{array}{l}\text { Reope- } \\
\text { raçóes }\end{array}$ & $\begin{array}{c}\text { Causas } \\
\text { de } \\
\text { Obbito }\end{array}$ \\
\hline $\begin{array}{l}\text { 1- AT } \\
\text { 2- DVEV + EP } \\
\text { 3-AP C/ SIVI }\end{array}$ & $\begin{array}{l}3 d \\
7 d \\
2 d\end{array}$ & $\begin{array}{l}5 \mathrm{~mm} \\
5 \mathrm{~mm} \\
5 \mathrm{~mm}\end{array}$ & $\begin{array}{l}\text { PTFE } \\
\text { PTFE } \\
\text { TPFE }\end{array}$ & $\begin{array}{l}\text { Obstrução } \\
\text { Obstrução } \\
\text { Arritmia }\end{array}$ & $\begin{array}{c}\text { B-T } \\
- \\
-\end{array}$ & $\begin{array}{l}\text { Hipóxia } \\
\text { Hipóxia } \\
\text { Morte } \\
\text { súbita }\end{array}$ \\
\hline $\begin{array}{l}\text { 4-T. Fallot + AP } \\
5-\mathrm{T} \text {. Fallot + AP } \\
\text { 6- AP c/ SIVI }\end{array}$ & $\begin{array}{l}8 \mathrm{~m} \\
1 \mathrm{a} \\
1 \mathrm{~m}\end{array}$ & $\begin{array}{l}5 \mathrm{~mm} \\
5 \mathrm{~mm} \\
5 \mathrm{~mm}\end{array}$ & $\begin{array}{l}\text { PTFE } \\
\text { PTFE } \\
\text { PTFE }\end{array}$ & $\begin{array}{l}\text { Obstrução } \\
\text { Obstruçăo } \\
\text { Isquemia } \\
\text { miocárdica }\end{array}$ & $\begin{array}{c}B-T \\
B-T \\
-\end{array}$ & $\begin{array}{l}\text { Hipóxia } \\
\text { Hipóxia } \\
\text { Morte } \\
\text { súbita }\end{array}$ \\
\hline $\begin{array}{l}7 \text { - Isomerismo } \\
\text { 8- DVEV + EP }\end{array}$ & $\begin{array}{r}6 d \\
20 d\end{array}$ & $\begin{array}{l}5 \mathrm{~mm} \\
5 \mathrm{~mm}\end{array}$ & $\begin{array}{l}\text { PTFE } \\
\text { PTFE }\end{array}$ & $\begin{array}{l}\text { Obstruçāo } \\
\text { Sepsis }\end{array}$ & $\dot{-}$ & $\begin{array}{l}\text { Hipoxia } \\
\text { Insuf. } \\
\text { Respiratória }\end{array}$ \\
\hline
\end{tabular}

AT: atresia tricusside; DVEV: dupla via de entrada ventricular; EP: estenose pulmonar; AP c/ SIVl: atresia pulmonar com septo interventricular integro; T. Fallot: tétrade de Fallot; B-T: Blalock-Taussig. PTFE: Polytetrafluporethylene. 
MALUF, M. A.; ANDRADE, J. C. S.; CARVALHO, A.; CATANI, R.; VEGA, H.; ANDRADE, J. L.; SILVA, C.; CARVALHO, W. B.; BUFFOLO, E. - Operaçâo de Blalock-Tassig modificada para o tratamento paliativo de cardiopatias congênitas com hipofluxo pulmonar. Rev. Bras. Cir. Cardiovasc., 10 (3): 126-132, 1995.

Dentre as intercorrências, destacam-se:

- Intra-operatórias: Quatro $(5,5 \%)$ crianças apresentaram bradicardia e hipóxia temporárias durante o procedimento, melhorando com hiperventilaçāo e uso de drogas inotrópicas.

- Pós-operatórias: Seis $(8,3 \%)$ crianças apresentaram sangramento espontâneo nas primeiras horas de PO, melhorando após administração de protamina e plasma fresco. Em nenhum caso toi necessária a reintervençăo para a revisão da hemostasia.

As complicações pulmonares mais frequeentes foram: atelectasia pulmonar $8(11,1 \%)$ casos, infecção pulmonar $6(8,3 \%)$ casos, derrame pleural 5 $(6,9 \%)$ casos, porém com apenas 1 obito (Tabela 5).

TABELA 5

INTERCORRENNCIAS NO PÓS-OPERATÓRIO IMEDIATO

\begin{tabular}{lccccc}
\hline $\begin{array}{l}\text { Tipo de } \\
\text { Intercorrências }\end{array}$ & Pacientes & \multicolumn{2}{c}{$\begin{array}{c}\text { Evoluçāo } \\
\text { Pós- } \\
\text { Operatória } \\
\text { Bom }\end{array}$} & Óbito \\
\hline Sangramento & 6 & $(08,3)$ & 2 & - \\
Atelectasia Pulmonar & 8 & $(11,1)$ & 8 & - \\
Infecçāo Pulmonar & 6 & $(08,3)$ & 5 & 1 \\
Derrame Pleural & 5 & $(06,9)$ & 5 & - \\
Lesăo Nervo Frênico & - & & - & - \\
Hiperfluxo Pulmonar & - & & - & - \\
Transudaçăo da Prótese & - & & - & - \\
PTFE & & & & & \\
\hline
\end{tabular}

\section{COMENTÁRIOS}

A operação de Blalock-Taussig modificada, constitui uma excelente escolha no tratamento paliativo de cardiopatias congênitas com hipofluxo pulmonar.

Esta operação pode beneficiar desde neonatos gravemente doentes, até portadores de cardiopatias complexas, ou constituir operaçăo definitiva, naqueles casos onde não é possivel esse tipo de correçăo cirúrgica.

O uso de prótese para realizar a derivação sistêmico-pulmonar tem permitido um adequado fluxo pulmonar, melhorando o quadro clínico do paciente e favorecendo o desenvolvimento da árvore arterial pulmonar. Por estes motivos esta técnica foi muito difundida e empregada pela maioria dos grupos que trabalham nesta área, com bons resultados tardios 4,6, 9-12; no entanto, a operação de BlalockTaussig usando a artéria subclávia (BT clássico) continua sendo a operação preferida por alguns autores $2,13,14$.

Sem lugar a dúvidas, a prótese de PTFE é a mais correntemente usada pelos grupos que realizam esta operação de rotina $1,4,6,10$. Na nossa casuística a prótese de PTFE foi empregada em 69 $(94,5 \%)$ casos.

Próteses biológicas preservadas têm sido usadas com menor freqüência como: veia umbilical ${ }^{11,12}$ ou artéria mamária bovina 1,2,12-15, com bons resultados tardios.

Em relação ao diâmetro da prótese, existe o consenso de que o desvio do sangue năo depende do calibre da mesma e sim do orificio da artéria subclávia ou tronco braquiocefálico, que atua como regulador do fluxo ${ }^{6}$. 0 diâmetro de $5.0 \mathrm{~mm}$ tem sido a escolha da maioria dos autores, porém diâmetros de $4.0 \mathrm{~mm}$ mostraram bons resultados 15 .

$\mathrm{Na}$ nossa casuística usamos próteses de 4.0 $\mathrm{mm}$ em apenas 2 pacientes, devido a grave hipoplasia das artérias pulmonares.

A presença de anomalias de origem dos vasos do arco aórtico, ou a própria hipoplasia da artéria subclávia poderá ser um fator limitante nos resultados desta operaçăo. É por isto que, desde $1987^{12}$, preferimos realizar anastomose da prótese com o TBC, facilitando a confecção da sutura e garantindo melhor fluxo para o pulmão.

$\mathrm{Na}$ nossa série, $24(34,7 \%)$ neonatos foram operados com esta técnica. Nesta série houve 2 óbitos, nenhum relacionado à perviabilidado da derivaçāo (Tabela 3 ).

A preferência de uma toracotomia direita ou esquerda para realizar a operação não obedece a critérios rígidos e sim a preferéncia ou hábito do cirurgiāo. Justifica-se esta escolha quando há razōes anatômicas especiais como: aorta descendo à direita, anomalias de origem da artéria subclávia, presença de estenose nos ramos pulmonares, falta de confluència das artérias pulmonares, presença de artérias brônquicas suprindo a circulaçăo pulmonar, necessidade de realizar uma valvotomia pulmonar etc.

Nossa tendência foi realizar a derivaçāo do lado direito $(83,3 \%)$, especialmente tratando-se de neonatos $(45,8 \%)$ sendo usado nestes últimos o TBC $(75,7 \%)$.

Houve algumas intercorrências intra-operatórias provocadas pelo manuseio de estruturas próximas ao canal arterial (hipóxia, bradicardia), porém contornadas com hiperventilação e uso de drogas inotrópicas.

O uso de heparina em dose única $(1 \mathrm{mg} \mathrm{kg}$ 
MALUF, M. A.; ANDRADE, J. C. S.; CARVALHO, A.; CATANI, R.; VEGA, H.; ANDRADE, J. L.; SILVA, C.; CARVALHO, W. B.; BUFFOLO, E. - Operaçăo de Blalock-Tassig modificada para o tratamento paliativo de cardiopatias congénitas com hipofluxo pulmonar. Rev. Bras. Cir. Cardiovasc., 10 (3): 126-132, 1995.

peso) antes do pinçamento dos vasos constitui atitude aceita por todos os grupos, evitando a formaçăo de trombos que, eventualmente, poderiam embolizar as artérias pulmonares. Porém, a manutençăo da heparinizaçăo ou uso de antiagregantes plaquetários nos primeiros meses de pós-operatório divide as opiniōes, Nosso trabalho mostra uso de dose única de heparina, neutralizada com protamina em apenas 2 casos que apresentaram sangramento espontâneo.

Nesta série de pacientes, não tivemos intercorrências relacionadas a lesão do nervo frênico (paresia ou paralisia), reoperaçőes devidas a sangramentos ou sinais de hiperfluxo pulmonar.
Tivemos $5(6,9 \%)$ óbitos devidos a obstrução da prótese no pós-operatório imediato, sendo que 3 foram submetidos a nova intervenção, sem sucesso. Este incidente aconteceu nos primeiros 22 casos desta série, mas não foi registrado nos seguintes 50 casos consecutivos.

A operaçāo de Blalock-Taussig, transcorridos 50 anos de sua concepção, continua sendo uma opçăo tática para conseguir uma imediata melhora de crianças com grave comprometimento do seu fluxo pulmonar. É uma operaçăo simples, rápida, que exige depurada e cuidadosa técnica cirúrgica, podendo ser realizada com baixa incidência de intercorrências e alta taxa de sobrevida.

MALUF, M. A.; ANDRADE, J. C. S.; CARVALHO, A.; CATANI, R.; VEGA, H.; ANDRADE, J. L.; SILVA, C.; CARVALHO, W. B.; BUFFOLO, E. - Blalock-Taussig operation for palliative treatment of congenital heart disease with low pulmonary flow. Rev. Bras. Cir. Cardiovasc., 10 (3): 126-132, 1995.

ABSTRACT: From January 1990 to November 1994, 72 patients with congenital heart defects and low pulmonary flow underwent modified Blalock-Taussig produce. There were $44(61.1 \%)$ males and 28 $(38.8 \%)$ females with ages ranging from 2 days to 11 years (average 9 months). Thirty eight $(52,8 \%)$ patients had Tetralogy of Fallot: $7(9.7 \%)$ had pulmonary atresia with intact ventricular septum (PA/IVS); $6(8.4 \%)$, had transposition of the great arteries (TGA) with pulmonary stenosis (PS): $6(8,4 \%)$ had tricuspid atresia (TA) with PS; $6(8.4 \%)$ had double inlet of right ventricle (RV) or left ventricle (LV) and PS; $3(4.2 \%)$ had corrected transposition of the great arteries (CTGA) with ventricular septal defects (VSD) and PS; 2 $(2.7 \%)$ had double outlet of RV or LV and PS; $2(2.7 \%)$ had atrio-ventricular canal defects (A-VC) and PS; $2(2.7 \%)$ patients had right or left Isomerism and PS. The decision to surgical indication was based on: a) new borns with "ductus dependent" heart defects; b) lactents with important cianosis or hypoxia; $c$ ) infants with heart defects without possibilities of biventricular correction. The surgical technique employed was the Blalock-Taussig operation using 4 or $5 \mathrm{~mm}$ Polytetrafluoroethy (PTFE) prosthesis in 69 (94.5\%) cases, umbilical vein in $3(4.1 \%)$ cases and bovine thoracic artery in $1(1.4 \%)$ case. Before the arteries were clamped $1 \mathrm{mg} / \mathrm{kg}$ of heparine was given without mobilization, with protamine, after the procedure. During the post-operative period, anticoagulants were not given. The prosthesis obstruction was the main cause of death and was related to artery anatomy: subclavian and pulmonary artery diameter and/or problems with the technique. The modified Blalock-Taussig operation showed itself to be a reliable palliative treatment to heart defects with low pulmonary flow.

DESCRIPTORS: Blalock-Taussig operation, modified. Heart defects, congenital, Blalock-Taussig operation. Heart defects, congenital, low pulmonary flow, surgery. 
MALUF, M. A.; ANDRADE, J. C. S.; CARVALHO, A.; CATANI, R.; VEGA, H.; ANDRADE, J. L.; SILVA, C.; CARVALHO, W. B.; BUFFOLO, E. - Operaçăo de Blalock-Tassig modificada para o tratamento paliativo de cardiopatias congênitas com hipolluxo pulmonar. Rev. Bras. Cir. Cardiovasc., 10 (3): 126-132, 1995.

\section{REFERĖNCIAS BIBLIOGRÁFICAS}

1 AMATTO, J. J.; MARBEY, M. L.; BUSH, C.; GALDIERI, R. J.; COTRONEO, J. V.; BUSHONG, J. - Systemicpulmonary polytetrafluorethylene shunts in palliative operations for congenital disease: revival of the central shunt. J. Thorac. Cardiovasc. Surg., 95: 62-69, 1988.

ARCIENAGAS, E.; FARROKI, Z, Q.; HAKIMI, M.; PERRY, B. L.; GREEN, E. W. - Classic shunting operations for congenital cyanotic heart defects. $J$. Thorac. Cardiovasc. Surg., 84: 88-96, 1982.

BLALOCK, A. \& TAUSSIG, H. - Surgical treatment of malformations of the heart in which there is pulmonary stenosis or pulmonary atresia. JAMA, 128: 189-202, 1945.

4 BOVE, E. L.; KOHMAN, L.; SEREIKA, S.; BYRUM, C. J.: KAVEY, R. E. W.: BLACKMAN, M. S.; SONDHEIMER, H. M.; ROSENTHAL, A. - The modified Blalock-Taussig shunt: analysis of adequacy and duration of palliation. Circulation, 76 (Supl 3): 19-23, 1987.

CIARAVELLA, J. M. \& MIDGLEY, F. M. - Construction of interposition polytetrafluoroethylene ascending aorta-pulmonary artery shunt. Ann. Thorac, Surg., 26: $570-572,1980$.

6 DE LEVAL, M. R.; MCKAY, R.; JONES, M.; STARK, J.; MACARTNEY, F. J. - Modified Blalock-Taussig shunt: use of subclavian artery orifice as flow regulator in prosthetic systemic-pulmonary artery shunts. J. Thorac. Cardiovasc. Surg., 81: 112129, 1981.

7

GAZZANIGA, A. B.; ELLIOT, M. P.; SPERLING, D. R. DIETRICK, W. R.; EISENMAN, J. I.; MCRAE, D. M.: BARTLETT, R. H. - Microporous expanded polytetrafluoroethylene arterial prosthesis for construction of aortopulmonary shunts: experimental and clinical results. Ann. Thorac. Surg., 21: 322-327, 1976.
8 GUYTON, R. A.; OWENS, J. E.; WAUMETT, J. D.; DOOLEY, K. J.; HATCHER, C. R.; WILLIAMS, W. H. - The Blalock-Taussig shunt: low risk, effective palliation, and pulmonary artery growth. J. Thorac. Cardiovasc. Surg., 85: 917-922, 1983.

9 ILBAWI. M. N.; GRIECO, J.; DeLEON, S. Y.; IDRISS F. S.; MUSTER, A. J.; BERRY, T. E.; KLICH, J. Modified Blalock-Taussig shunt in newborn infants. J. Thorac. Cardiovasc. Surg., 88: 770-775, 1984.

10 LAMBERTI, J. J.; CARLISLE, J.; WALDMAN, J. D.; LODGE, F. A.; KIRKPATRICK, S. E.; GEORGE, L.; MATHEWSON, J. W.; TURNER, S. W.; PAPPELBAUM, S. J. - Systemic-pulmonary shunts in infants and children: early and late results. $J$. Thorac. Cardiovasc. Surg., 88: 76-81, 1984.

11 LEĀO, L. E. V.; ANDRADE, J. C. S.; SUCCI, J. E.; CUEVA, C. C.; RIBEIRO, E. E.; CARVALHO, A. C. C.; BUFFOLO, E. - Modified Blalock-Taussig shunt with an umbilical vein graft. Texas Heart inst. $J$, 12: $65-71,1985$

12 MALUF, M.; ANDRADE, J. C.; CATANI, R.; CARVALHO, A. C.; LIMA, W. C.; ANDRADE, J. L.; LEĀO. L. E. V.; BUFFOLO, E. - Derivação sistêmico-pulmonar em cardiopatias congênitas com hipofluxo pulmonar: análise critica da técnica cirúrgica e resultados imediatos. Arq. Bras. Cardiol., 56: 281. 286, 1991.

13 STEWART, S.; ALEXON, C.; MANNING, J.- Long-term palliation with the classic Blalock-Taussig shunt. J. Thorac. Cardiovasc. Surg., 96: 117-121, 1988.

14 ULLOM, R. L.; CRAWFORD, F. A.; ROSS, B. A.; SPINALE, F. - The Blalock-Taussig shunt in infants: standard versus modified. Ann. Thorac. Surg., 44: 539-543, 1987

15 VOUHE, P. R.; CALIANI, J; TAMISIER, D. - Systemicpulmonary shunts using a preserved bovine internal mammary artery. In: Proceedings of Bioflow Small Diameter Graft Symposium-Garmisch 1989. St. Paul, Biovascular Inc., 1989, p. 23-30. 